\title{
World tuberculosis day 2017
}

\section{Editorial}

"Unite to end TB" is still the theme of this year's World Tuberculosis Day. This annual event commemorates the date March 24th in 1882 when Dr. Robert Koch announced his discovery of Mycobacterium tuberculosis (M.tb), the bacillus that caused tuberculosis (TB). Misdiagnosis of TB remains and several health care professionals often do not "think TB". World Tuberculosis Day is designed to raise the common public awareness about this epidemic disease as well as get their efforts for totally eradication of this disease that approximately one-and-a-half million deaths occur each year. Various World Tuberculosis Day activities and events are organized by various organizations involved in the Stop TB Partnership. Campaign activities include charity events to raise funds for TB control in countries that need assistance, community discussion groups that are organized to look at ways to prevent TB, award ceremonies or other events to honor the life and work of those who dedicate their lives to fight against TB, and photo exhibitions that showcase images to raise global awareness of TB. In 1996, the World Health Organization (WHO) joined the International Union against Tuberculosis and Lung Disease (IUATLD) and other organizations to promote World Tuberculosis Day. The Stop TB Partnership, called the Stop TB Initiative at the time of its inception, was established in 1998. It is a network of countries and organizations fighting TB. TB, the leading infectious cause of death globally, found in every country in the world; far surpassing both HIV and malaria. Successfully reduced morbidity and mortality rates from HIV/AIDS and malaria are due to huge investments into these two diseases. The WHO estimates that two billion people (one third of the world's population) are infected with M.tb. Each year, about 10.4 million people fall ill from TB. Due to an airborne disease, TB can be spread by coughing or sneezing. $\mathrm{TB}$ is responsible for the cycle of poverty and illness that entraps families, communities, and even entire countries, that contribute to economic devastation. Among the most vulnerable are children, women, and those with HIV/AIDS. Each year, there are about 580,000 cases of multidrug-resistant TB (MDR-TB) that is growing resistant to currently available drugs. This disease is becoming more deadly and difficult to treat. The globally growing problem of drug resistance, major shortfalls in the available resources, potential long latency periods, unrecognized primary and re-infections, relatively asymptomatic disease onset leading to transmission months before most patients present for clinical care, a huge unrecognized reservoir of latent TB infections compounded by a limited repertoire of safe and cheap TB drugs, and lengthy treatment with multiple drugs contributing to poor compliance are important factors that distinguish TB from HIV/AIDS and malaria, in addition to the poorly understood complex biology and pathogenesis of M.tb infection.

"End TB" strategy aims to reduce new TB cases by $90 \%$ and reduce TB deaths by $95 \%$ between 2015 and 2035 , and ensure that no
Volume 4 Issue I - 2017

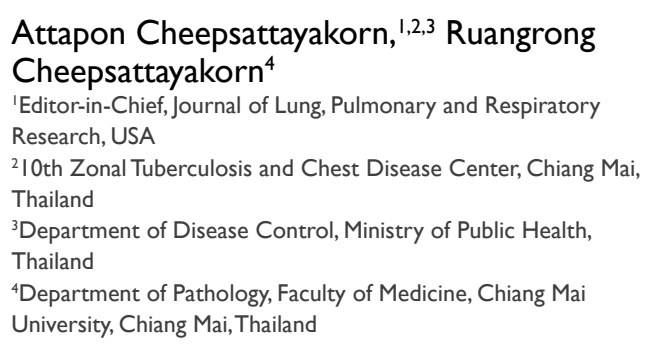

Correspondence: Attapon Cheepsattayakorn, I0th Zonal Tuberculosis and Chest Disease Center, I 43 Sridornchai Road Changklan Muang Chiang Mai 50I00 Thailand,Tel 6653 I40767, 6653 276364, Fax 6653 140773, 6653 273590, Email Attapon1958@gmail.com, attaponche@yahoo.com

Received: March 12, 2017| Published: March 15, 2017

family burdened with catastrophic expenses due to TB. Nevertheless, this strategy seems unrealistic to achieve in a situation where more than a million cases of MDR-TB $(52 \%, 2016)$ occur every year, only $20 \%$ of these cases can access appropriate care and treatment outcome remain unsatisfactory. Additionally, XDR-TB has been identified in more than 100 countries. A recent mathematical model study revealed that MDR-TB may sustain epidemic spread, even in the presence of some fitness cost and over time potentially replace drug susceptible strains in the absence of effective MDR-TB control programs.

\section{Conclusion}

It is clearly known that research funding allocated to TB is substantially less than for HIV/AIDS and malaria. No effective TB vaccines, the high death rates associated with MDR-TB/XDR-TB, the lengthy treatment duration of TB, the long-term functional disability suffered by TB patients due to the associated drug toxicities and permanent lung damage, the large burden of comorbidity with TB and HIV/AIDS or non-communicable diseases, sparse affordable TB drug pipeline, and the neglect of childhood TB are among many problems that contribute to currently unacceptable status quo.

\section{Acknowledgements}

None.

\section{Conflict of interest}

The author declares no conflict of interest. 